\title{
A sustainable post-COVID future
}

\author{
Experts around the world have been informing governments' plans for a post-pandemic recovery. Leena Srivastava, \\ Deputy Director General for Science at the International Institute for Applied Systems Analysis (IIASA), and Heide \\ Hackmann, Chief Executive officer at the International Science Council (ISC), talk to Nature Sustainability about the \\ recent joint effort 'Bouncing forward sustainably. Pathways to a post-COVID world'.
}

\section{What was the impetus behind this col- laboration between IIASA and ISC? What was the aim?}

LS: While it was obvious early in 2020 that the world was unprepared for the system-wide challenges posed by the COVID-19 pandemic, it was also apparent that 2020 would be a year of desperate, yet feasible, innovations and actions implemented under duress. Some of these innovations and actions, with necessary refinement and support, could hold the key to the transformations urgently needed to achieve both our climate and sustainable development goals. IIASA and ISC were well placed to mobilize the science and policy-relevant communities to inform the selection of promising transformative actions and framework conditions that will be essential for their success.

HH: While this collaborative initiative is about essential system transformations within reach, sound scientific analysis and engagement - focused on ensuring a more equitable and resilient development would greatly support the hands of decision makers in being able to implement those transformations at the scale and speed necessary to meet multiple global challenges. ISC and IIASA are both committed to supporting systemic sustainability transformations and make natural partners in this effort.

The synthesis report published in January 2021 emphasizes the need for 'systemic transformative changes'. Where do we need them the most? What are the main barriers to enable them?

LS: A select list of systemic transformative changes identified in this initiative, that could lay the foundation for much wider sustainability transformations, would include those in: (1) governance systems - at global and national levels - to achieve system-level coordinated actions yielding multiple dividends and minimizing trade-offs for sustainable development;

(2) science systems - including setting research agendas; defining incentive systems for researchers, in financial and recognition terms, to undertake systemic research in an open, collaborative manner;
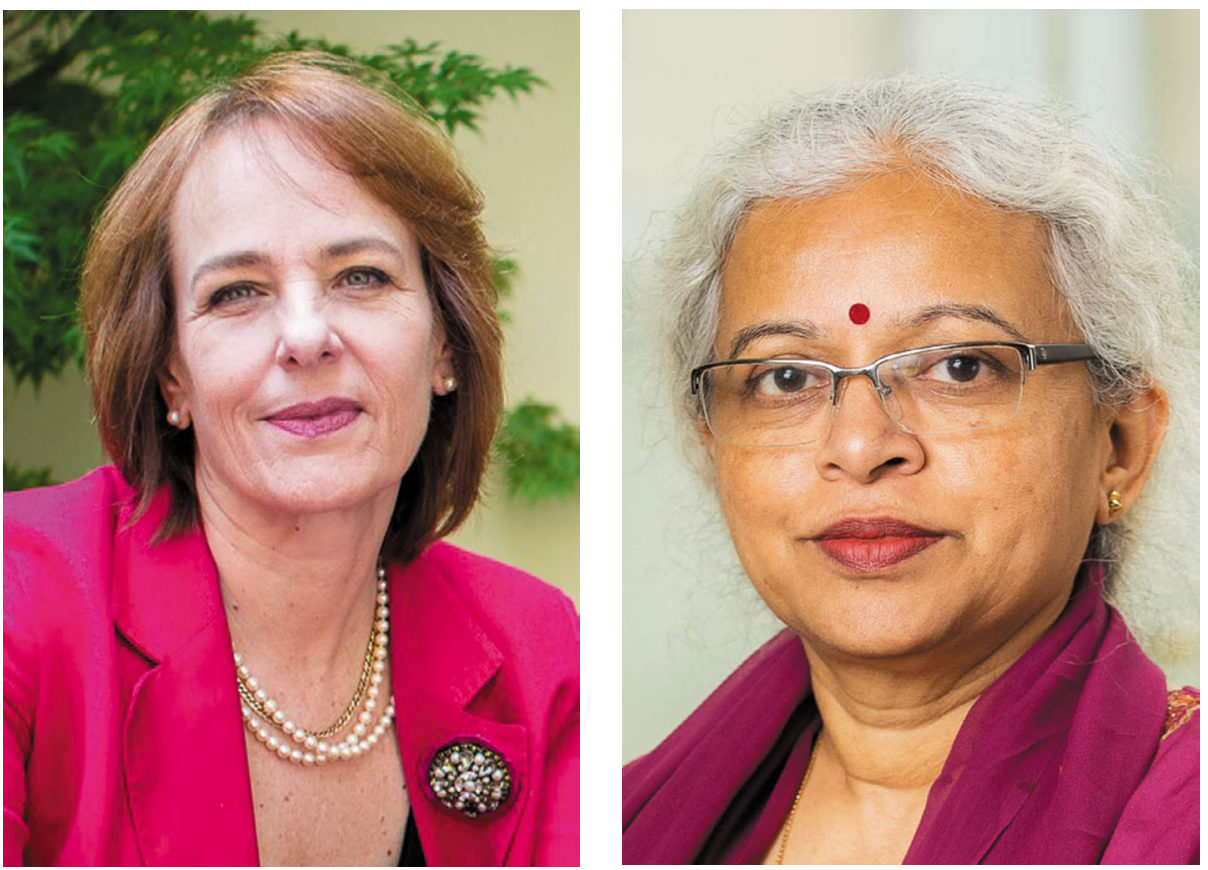

Heide Hackmann

as well as focusing on integrated, feasible solutions to global challenges; (3) urban areas towards highly digitally empowered, service-oriented, community-driven, resource-minimizing use of space. A number of these transformative changes could be effected quite cost-effectively through careful re-design of policies, regulations and incentives. The key barrier to achieving these transformations lies in our inability to think and act systemically due to structural barriers that exist at all levels - from education to business regulations.

HH: Our assessment was that while science systems responded quite well to the pandemic in general, there was substantial room for improvement. To deal better with future crises, researchers and scholars need to react more rapidly and be more agile (without lowering quality and whilst ensuring reliability), and be more responsive to the needs of policy makers and of citizens. To move science systems to a new frontier will require simultaneous changes in many
Leena Srivastava.

areas; many new policies and associated interventions. The report from this initiative provides a total of 38 recommendations. The imperatives of open, engaged science are key for the future.

Many experts recurrently mention the need to innovate institutions and so do you, yet the reality is one of entrenched practices. How do we move away from those and innovate?

LS: A most urgent need, to move away from entrenched practices, is to build a pervasive knowledge society, informed by integrated and trustworthy science, so as to effect confident, transformative changes at an institutional level. Continuous learning and skill upgradation based on knowledge responding to today's challenges, institutionalizing effective science-policy interfaces, and participatory approaches to decision making are all examples by which institutions can remain empowered, agile and responsive while bearing in mind local contexts. 
HH: The COVID-19 pandemic brought science to the centre of public attention and to policy making. Researchers and scholars - from diverse fields - engaged increasingly with the public and with policy makers. We need to build on this experience to develop more effective science-policysociety interactions and get researchers to recognize that going beyond just 'discovery' — incorporating communication and engagement with citizens and with policy makers - is valuable and necessary.

One of the messages from the report is the importance of equitable access to knowledge creation and dissemination. How do we achieve that? Who are the key players?

LS: Equitable access to knowledge between and within countries is a pre-condition for successful transformative changes leading to the three pillars of sustainable development. COVID-19 has sharply highlighted, once again, the need for all parts of the interconnected, complex and unequal world we live in, to move in step towards finding context-specific solutions designed in a bespoke manner - no silver bullets would work here, but international cooperation is key. Open and inclusive science is a global public good that needs the pro-active support of all governments.

HH: Adequate financing to overcome persistent global knowledge divides is essential. In addition, countries can to a considerable degree complement local scientific capacities through effective international collaboration and participation in global scientific networks of open knowledge exchange. Engaging other knowledge partners - including policy makers, practitioners, the business community and civil society - in those networks fosters mutual learning and problem solving in ways that enhance the socio-political legitimacy and, ultimately, the relevance of our research.

For systemic transformations, the report recommends more public-private partnerships - something many will agree on. Is this happening? At what scale is it more likely to work?

LS: The private sector is eager to engage with the sustainability agenda. It stepped up efforts both by itself and through unprecedented public-private partnerships to enable a rapid response to the pandemic emergency based on synergistic relationships. Today, we are faced with several other planetary emergencies, possibly of greater complexity and impact, that would benefit from the same level of private sector engagement. We need to learn from the spirit of the partnership that emerged throughout 2020 in order to support the transformative actions the IIASA-ISC initiative and others have identified - urban re-organization and re-purposing, for example, cannot succeed without a strong public-private partnership. HH: With few exceptions, the private sector makes the biggest contribution to national research budgets. Even with respect to fundamental research, in many countries, the private sector contributes more than the public sector and such contribution is growing. Harnessing the potential of the private sector is critical to achieving social goals. That was certainly evident in the response to the pandemic. Additionally, the private sector possesses data that are critical to developing effective responses to crises. Some companies have made valuable data available: for example, Google has shared so-called community mobility reports and Yandex, Russia's biggest IT company, has made data available that showed how people's mobility had changed in response to the COVID-19 pandemic (Russia Beyond 2020). ICT companies shared knowledge and made their technology platforms available for track and trace. However, these are exceptions. Much more needs to be done.

Now that the report has been published, what are the next steps?

LS: IIASA and ISC are preparing for the launch of Phase 2 of this initiative in June 2021, which will strengthen the recommendations from Phase 1 through deeper analysis as well as by broadening the menu of transformative opportunities being discovered as we continue to adapt to our experiences from 2020 and 2021. This phase of the initiative will run for two years. HH: Realizing 'transformations within reach' will require researchers and scholars to work closely with communities of influence and practice from different societal sectors. Phase 2 of the IIASA and ISC global consultative platform will convene such multi-stakeholder communities to catalyse the co-design of radical, urgent and tractable transformation scenarios and associated pathways, and ultimately advocate and advance their implementation.

Interviewed by Monica Contestabile

Published online: 15 March 2021

https://doi.org/10.1038/s41893-021-00703-9 